\title{
CHARACTERIZING SPIRAL ARM AND INTERARM STAR FORMATION
}

\author{
K. Kreckel ${ }^{1}$, G. A. Blanc ${ }^{2,3,4}$, E. Schinnerer ${ }^{1}$, B. Groves $^{5}$, A. Adamo ${ }^{6}$, A. Hughes ${ }^{7,8}$, and S. Meidt ${ }^{1}$ \\ ${ }^{1}$ Max Planck Institut für Astronomie, Königstuhl 17, D-69117 Heidelberg, Germany; kreckel@mpia.de \\ 2 Departamento de Astronomía, Universidad de Chile, Camino del Observatorio 1515, Las Condes, Santiago, Chile \\ ${ }^{4}$ Centro de Astrofísica y Tecnologías Afines (CATA), Camino del Observatorio 1515, Las Condes, Santiago, Chile
Visiting Astronomer, Observatories of the Carnegie Institution for Science, 813 Santa Barbara Street, Pasadena, CA 91101, USA \\ ${ }^{5}$ Research School of Astronomy and Astrophysics, Australian National University, Canberra, ACT 2611, Australia \\ ${ }^{6}$ Department of Astronomy, The Oskar Klein Centre, Stockholm University, AlbaNova University Centre, SE-106 91 Stockholm, Sweden \\ ${ }^{7}$ CNRS, IRAP, 9 Av. du Colonel Roche, BP 44346, F-31028 Toulouse cedex 4, France \\ ${ }^{8}$ Université de Toulouse, UPS-OMP, IRAP, F-31028 Toulouse cedex 4, France \\ Received 2016 March 25; revised 2016 May 28; accepted 2016 June 11; published 2016 August 11
}

\begin{abstract}
Interarm star formation contributes significantly to a galaxy's star formation budget and provides an opportunity to study stellar birthplaces unperturbed by spiral arm dynamics. Using optical integral field spectroscopy of the nearby galaxy NGC 628 with VLT/MUSE, we construct $\mathrm{H} \alpha$ maps including detailed corrections for dust extinction and stellar absorption to identify $391 \mathrm{H}$ II regions at $35 \mathrm{pc}$ resolution over $12 \mathrm{kpc}^{2}$. Using tracers sensitive to the underlying gravitational potential, we associate $\mathrm{H}$ II regions with either arm (271) or interarm (120) environments. Using our full spectral coverage of each region, we find that most physical properties (luminosity, size, metallicity, ionization parameter) of $\mathrm{H}$ II regions are independent of environment. We calculate the fraction of $\mathrm{H} \alpha$ luminosity due to the background of diffuse ionized gas (DIG) contaminating each $\mathrm{H}$ II region, and find the DIG surface brightness to be higher within $\mathrm{H}$ II regions than in the surroundings, and slightly higher within arm $\mathrm{H}$ II regions. Use of the temperature-sensitive $[\mathrm{S} \mathrm{II}] / \mathrm{H} \alpha$ line ratio instead of the $\mathrm{H} \alpha$ surface brightness to identify the boundaries of $\mathrm{H}$ II regions does not change this result. Using the dust attenuation as a tracer of the gas, we find depletion times consistent with previous work $\left(2 \times 10^{9} \mathrm{yr}\right)$ with no differences between the arm and interarm, but this is very sensitive to the DIG correction. Unlike molecular clouds, which can be dynamically affected by the galactic environment, we see fairly consistent properties of $\mathrm{H}$ II regions in both arm and interarm environments. This suggests either a difference in star formation and feedback in arms or a decoupling of dense star-forming clumps from the more extended surrounding molecular gas.
\end{abstract}

Key words: galaxies: individual (NGC 628) - galaxies: ISM - galaxies: spiral - galaxies: star formation - H II regions - ISM: structure

\section{INTRODUCTION}

Studies of extragalactic star formation are necessarily biased toward studying spiral arms, where both gas and star formation are concentrated. However, giant molecular clouds (GMCs) spend as long or even longer in the interarm region (Dobbs et al. 2006), and a significant fraction (30\%-60\%) of star formation in nearby galaxies is observed to occur in interarm regions (Foyle et al. 2010). This fraction drops (to $8 \%$ in M51, Lee et al. 2011; 14\% in M31, Azimlu et al. 2011) in studies that resolve individual $\mathrm{H}$ II regions, where the brightest $\mathrm{H}$ II region is almost an order of magnitude brighter in the arm than in the interarm. Some of this difference may be due to contamination of tracers of star formation on large $(\sim 500 \mathrm{pc})$ scales by emission from diffuse ionized gas (DIG), which makes up on average $\sim 60 \%$ of the total $\mathrm{H} \alpha$ flux of a galaxy (Rand 1992; Greenawalt et al. 1998; Zurita et al. 2000; Oey et al. 2007; Haffner et al. 2009).

Most existing studies comparing spiral arm and interarm H II regions are performed using $\mathrm{H} \alpha$ narrow-band imaging, and as such they are limited to an analysis of the luminosities and sizes of the H II regions without the ability to properly correct for varying contributions from [N II], dust extinction, or the DIG. Statistical studies of H II regions have found evidence for a steeper slope in the luminosity function for the interarm than for the spiral arms (Kennicutt \& Hodge 1980; Kennicutt et al. 1989; Rand 1992; Thilker et al. 2000; Scoville et al. 2001), but in many cases no difference has been observed (Knapen et al. 1993; Rozas et al. 1996; Knapen 1998; Gutiérrez et al. 2011). The changing slope is largely attributed to statistical effects, because spiral arms contain more H II regions and therefore more luminous regions (Knapen 1998), or increased blending of individual $\mathrm{H}$ II regions, as the arms have an overall higher surface density (Scoville et al. 2001). In their detailed study of H II regions in M31, Azimlu et al. (2011) found similar slopes at the bright end, but that the luminosity functions for arm and interarm regions peak at different luminosities, which they attribute to a large population of aged B stars in the interarm from a recent starburst.

Recent development of new optical integral field unit (IFU) spectrographs allows for measurement of intrinsic $\mathrm{H} \alpha$ emission from $\mathrm{H}$ II regions, including corrections for [N II], dust attenuation, stellar absorption, and DIG, while providing full spectral and 2D information. While most of these effects are directly constrained by well established methods using the additional spectral information, measuring the amount of $\mathrm{H} \alpha$ flux arising from the DIG is more uncertain. In addition to spatial identification via its more diffuse structure (Zurita et al. 2000), the DIG also exhibits distinctive spectral diagnostic line ratios (Madsen et al. 2006; Blanc et al. 2009). As the DIG morphology is expected to be complex, and to vary across environmental features, deep spectral mapping is necessary to rigorously isolate this contaminant of star formation studies. 
There are many large optical IFU galaxy surveys underway with kiloparsec scale resolution (CALIFA, Sánchez et al. 2012; MANGA, Bundy et al. 2015; SAMI, Bryant et al. 2015) that are well suited to studying bulk star formation in galaxies. The tight correlation between molecular gas and star formation on these scales suggests a universal star formation law (Schmidt 1959; Kennicutt 1989, 1998; Bigiel et al. 2008); however, detailed studies reveal an additional dependence on local conditions and environmental parameters (Leroy et al. 2013). As most $\mathrm{H}$ II regions have sizes between 10 and 100 pc (Azimlu et al. 2011; Gutiérrez et al. 2011; Whitmore et al. 2011), significant information is lost with spatial averaging. Nearby galaxies provide the only opportunity to explore the physics of star formation at the relevant scales of $<50 \mathrm{pc}$.

We explore the impact of spiral arms on galaxy evolution by fully characterizing the physical conditions of arm and interarm $\mathrm{H}$ II regions using VLT/MUSE optical IFU spectroscopy within the nearby galaxy NGC 628 at scales of $35 \mathrm{pc}$. While our imaging does not cover the full disk of the galaxy, it provides a first look at the statistical studies enabled by these new observing techniques, and allows us to explore the use of diagnostic line ratios to fully characterize the properties of $\mathrm{H} \mathrm{II}$ regions (metallicity, ionization parameter, DIG fraction). NGC 628 , a nearly face-on type SAc grand design spiral, has a global star formation rate (SFR) of $0.68 M_{\odot} \mathrm{yr}^{-1}$ (Calzetti et al. 2010) and a stellar mass of $3.6 \times 10^{9} M_{\odot}$ (Skibba et al. 2011). We assume a distance of $7.2 \mathrm{Mpc}\left(1^{\prime \prime}=35 \mathrm{pc}\right)$ and $R_{25}=5 ! 25$ (Kennicutt et al. 2011). After a brief description of the data (Section 2) and our derived properties of $\mathrm{H}$ II regions (Section 3), we quantify differences in these properties between arm and interarm including DIG contribution (Sections 4.1 and 4.2), and discuss the impact on the star formation law (Section 4.3) before summarizing in Section 5.

\section{DATA}

NGC 628 was observed using the Multi-unit Spectroscopic Explorer (MUSE; Bacon et al. 2010) at the Very Large Telescope (VLT) in three positions (programmes ID 094.C0623 and ID 095.C-0473) centered on interarm regions (Figure 1). MUSE provides a $1^{\prime} \times 1^{\prime}$ field of view with 0 ". 2 pixel size. Observations were taken using the extended wavelength setting, covering 4650-9300 А, with 0 ". 8 seeing. Two northern and one southern pointings were observed with exposures of $845 \mathrm{~s}$ and $990 \mathrm{~s}$, respectively, in three $90^{\circ}$ rotations, alternated with separate sky exposures. All reductions were carried out using the standard ESO pipeline version 1.2.1. Emission lines $\mathrm{H} \beta \lambda 4861$, [O III] $\lambda 5007, \mathrm{H} \alpha \lambda 6563$, [N II] $\lambda 6548,[\mathrm{~N}$ II] $\lambda 6584,[\mathrm{~S} \mathrm{II}] \lambda 6717$, and [S II] $\lambda 6731$ are fit using LZIFU (Ho et al. 2016), and we tie all line kinematics and use MIUSCAT templates (Vazdekis et al. 2012) for the underlying stellar continuum. The typical spectral resolution for all lines fit $(<7000 \AA)$ is $\sim 2.75 \AA$ $\left(150 \mathrm{~km} \mathrm{~s}^{-1}\right)$. We reach a $3 \sigma$ surface brightness sensitivity for $\mathrm{H} \alpha$ of $1.5 \times 10^{-17} \mathrm{erg} \mathrm{s}^{-1} \mathrm{~cm}^{-2} \operatorname{arcsec}^{-2}$.

In the $\mathrm{H} \alpha$ map (not corrected for extinction) we geometrically identify $\mathrm{H}$ II region candidates using the 2DClumpfind algorithm (Williams et al. 1994), which finds local peaks in emission and extends them to lower flux levels. Peaks are identified using a lowest level at $25 \sigma$ $\left(1.3 \times 10^{-16} \mathrm{erg} \mathrm{s}^{-1} \mathrm{~cm}^{-2} \operatorname{arcsec}^{-2}\right)$ with contour steps of $2 \sigma$. This was found to provide thresholds sufficient to divide clustered areas and select isolated regions. We omit as foreground star candidates the $\mathrm{H}$ II regions with more than four pixels where the stellar velocity is $300 \mathrm{~km} \mathrm{~s}^{-1}$ less than systemic for NGC 628 . Using the $\mathrm{H} \alpha$ surface brightness map, we identify $391 \mathrm{H}$ II regions (Figure 1). We correct for dust attenuation using the $\mathrm{H} \alpha / \mathrm{H} \beta$ Balmer line decrement, assuming a Milky Way dust extinction law (Cardelli et al. 1989) and $R_{V}=3.1$, and we measure luminosities $10^{36.1} \mathrm{erg} \mathrm{s}^{-1}<L_{\mathrm{H} \alpha}<10^{39.0} \mathrm{erg} \mathrm{s}^{-1}$ (Figure 2), with most below the break typically observed at $L_{\mathrm{H} \alpha}=10^{38.7} \mathrm{erg} \mathrm{s}^{-1}$ (Kennicutt et al. 1989). All results are robust to variations in the exact $\mathrm{H} \alpha$ boundaries identified.

Lower temperatures in the $\mathrm{H}$ II regions than in the DIG result in a decreased $[\mathrm{S} \mathrm{II}] / \mathrm{H} \alpha$ line ratio (Madsen et al. 2006) and provide an alternative diagnostic for identifying $\mathrm{H}$ II regions. Using Clumpfind on an [S II] $/ \mathrm{H} \alpha$ map, we identify $319 \mathrm{H} \mathrm{II}$ regions (Figure 1, bottom), excluding foreground star candidates. Here we have applied 1" Gaussian smoothing, equivalent to the seeing, because the lower signal-to-noise ratio in [S II] results in irregular boundaries. Equivalent smoothing of the $\mathrm{H} \alpha$ line map does not significantly change the boundaries identified by $\mathrm{H} \alpha$ or the sizes of $\mathrm{H}$ II regions. As the $\mathrm{H}$ II regions are characterized by low [S II]/H $\alpha$ values, Clumpfind was applied to an inverted map (multiplied by -1 ). Peaks are identified using levels from 0.10 , consistent with the expected line ratio of a pure $\mathrm{H}$ II region (Madsen et al. 2006), with contour steps of 0.025 in the flux ratio, to a limiting level at 0.25 . Because the flux ratio is decoupled from the line intensities, we found this step size to be the minimum we could use that still resulted in reasonable division of clustered regions. However, in some regions this is insufficient to divide clearly distinct peaks (see Figure 1). Our level corresponding to the outer extent of the identified $\mathrm{H}$ II regions was chosen to balance the observed thresholds with the decreasing signal in the [S II] line. These boundaries are thus somewhat irregular, reflecting mainly the decreased line strength.

As we wish to compare star formation across environments, it is important to use a spiral arm tracer directly linked to the underlying gravitational potential and not one biased by the star formation itself. We use the mask from Foyle et al. (2010), who use a smoothed $3.6 \mu \mathrm{m}$ map to locate the old stellar population and Fourier decompose (work was done as part of Foyle et al.) the resulting image. However, their mask is limited to the central $2^{\prime}$ and excludes the central $45^{\prime \prime}$ of the galaxy. Because bulk molecular gas emission is strongly influenced by the gravitational potential (Colombo et al. 2014), we extend the spiral arms using HERACLES CO(2-1) maps (Leroy et al. 2009) at 14" $(500 \mathrm{pc})$ resolution (Figure 1$)$. Using the $\mathrm{H} \alpha$ boundaries, we find $271 \mathrm{H}$ II regions on the spiral arm and 120 in the interarm. Using the [S II] $/ \mathrm{H} \alpha$ boundaries, we find $192 \mathrm{H}$ II regions on spiral arms and 127 in the interarm. Fewer regions are detected in the arm with the latter method because it is less effective at dividing highly clustered H II complexes (see Section 4.2).

\section{RESULTS}

Our IFU observations provide spectral coverage for a large number of $\mathrm{H}$ II regions over their full spatial extent. This allows us to go beyond the parameters obtained in imaging studies (luminosities, sizes) to a full characterization of the physical conditions. In particular, we can more accurately account and correct for diffuse contribution to $\mathrm{H} \alpha$ emission, and test the assumptions traditionally used in these corrections. 

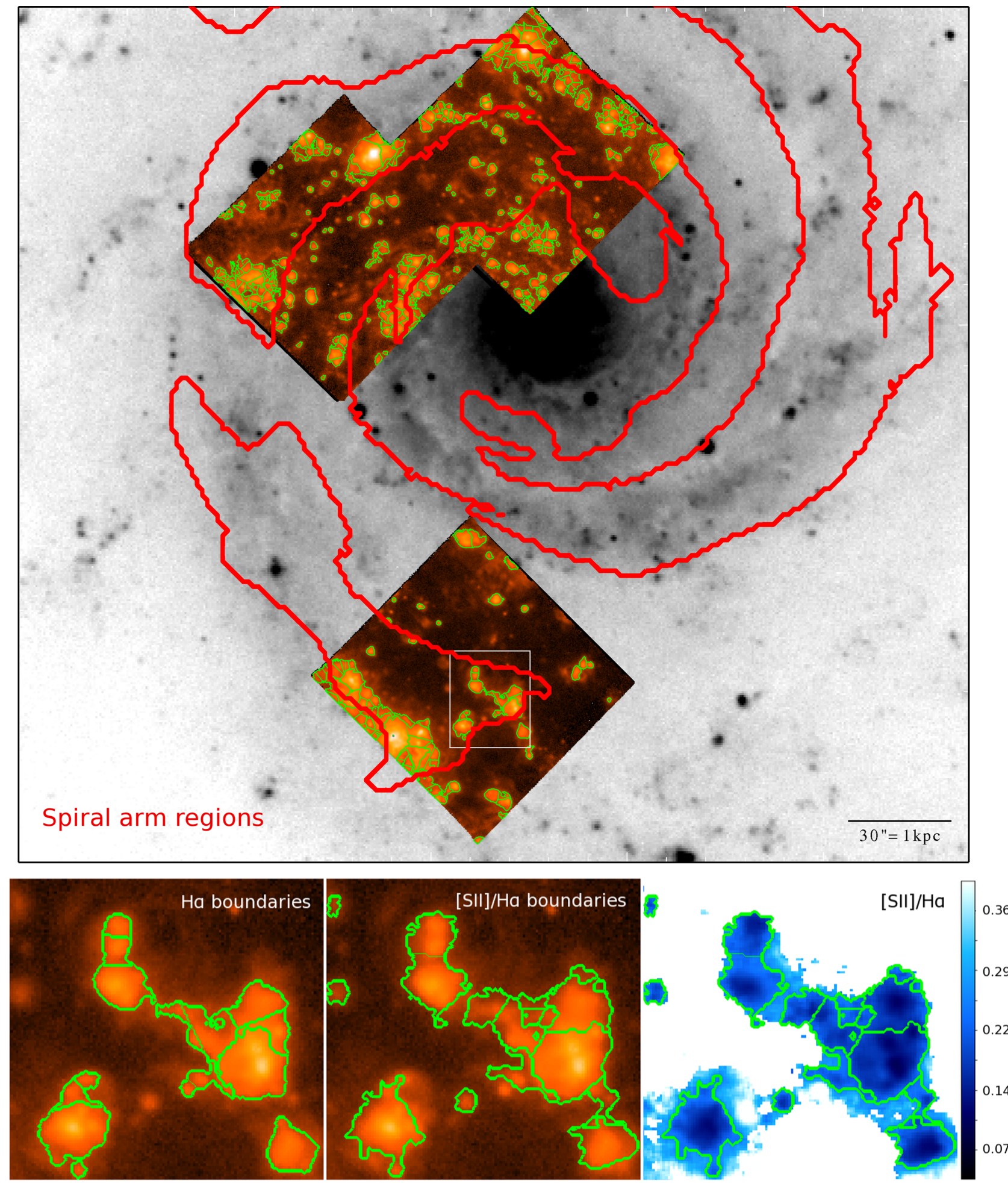

0.36

0.29

0.22

0.14

0.07

Figure 1. Top: $V$-band image of NGC 628 with the $\mathrm{H} \alpha$ maps for the three MUSE fields overlaid, with contours for the spiral arms (red). The use of Clumpfind on $\mathrm{H}$ II regions identified by $\mathrm{H} \alpha$ (green) cleanly selects isolated $\mathrm{H}$ II regions and breaks up more crowded $\mathrm{H}$ II complexes into individual regions. Bottom: a sample comparison (area in white box, above) of the $\mathrm{H}$ II regions identified using $\mathrm{H} \alpha$ line flux (left) or the $[\mathrm{S} \mathrm{II]} / \mathrm{H} \alpha$ line ratio (center, right) to define the boundaries of the region. $\mathrm{H}$ II regions identified by $[\mathrm{S} \mathrm{II}] / \mathrm{H} \alpha$ generally cover the same extent, and this method more easily identifies fainter regions but is not as good at dividing clustered regions.

\subsection{H II Region Properties}

We present in Figures 2 and 3 a comparison of the properties of $\mathrm{H}$ II regions for arm and interarm regions, using both methods of identifying $\mathrm{H}$ II regions. The results are similar for the two methods, so we focus here on the arm/interarm comparison using the $\mathrm{H} \alpha$ boundaries and discuss differences between the two methods in Section 4.2.

The range of luminosities of $\mathrm{H}$ II regions measured appears similar in the two environments (a Kolmogorov-Smirnov test 

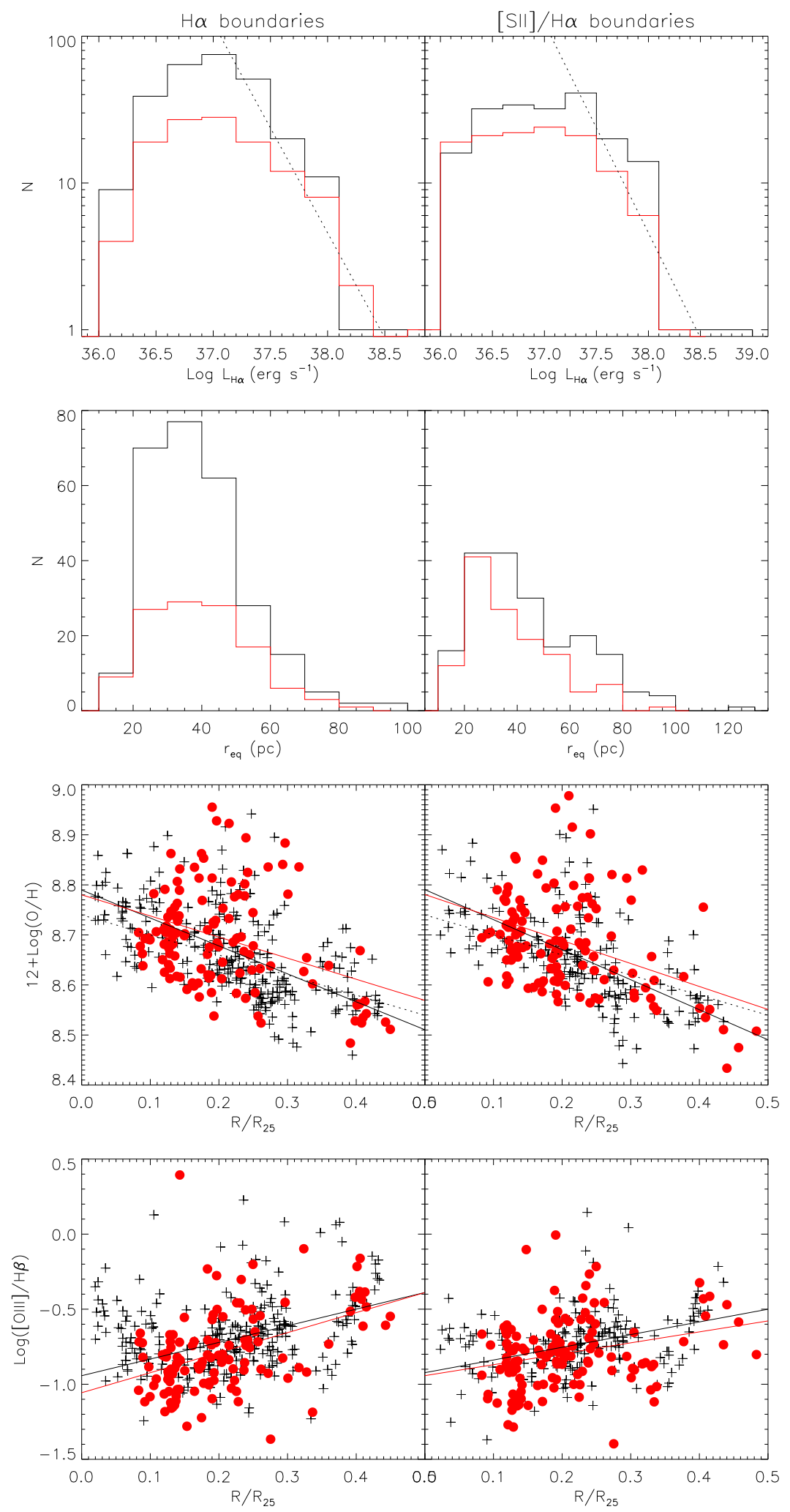

Figure 2. Comparison of properties of $\mathrm{H}$ II regions from the arm (black) and interarm (red) regions, using $\mathrm{H}$ II regions identified via $\mathrm{H} \alpha$ intensity (left) or [S II]/H $\alpha$ line ratios (right). Top panel: the range of luminosities of $\mathrm{H}$ II regions measured is similar between environments, and has a shallower slope than previous work (dotted line: Kennicutt \& Hodge 1980). Upper middle panel: the distribution of $r_{\mathrm{eq}}$ is also similar between environments, but depends strongly on the method of identifying the $\mathrm{H}$ II region. Lower middle panel: the metallicity follows the established radial gradient (dotted line: Berg et al. 2015); the typical uncertainty is 0.1 dex. Bottom panel: $[\mathrm{O} \mathrm{III}] / \mathrm{H} \beta$, which correlates with the ionization parameter, also varies radially; the typical uncertainty is 0.1 dex. No significant difference is seen in these parameters when comparing the arm and interarm regions. 


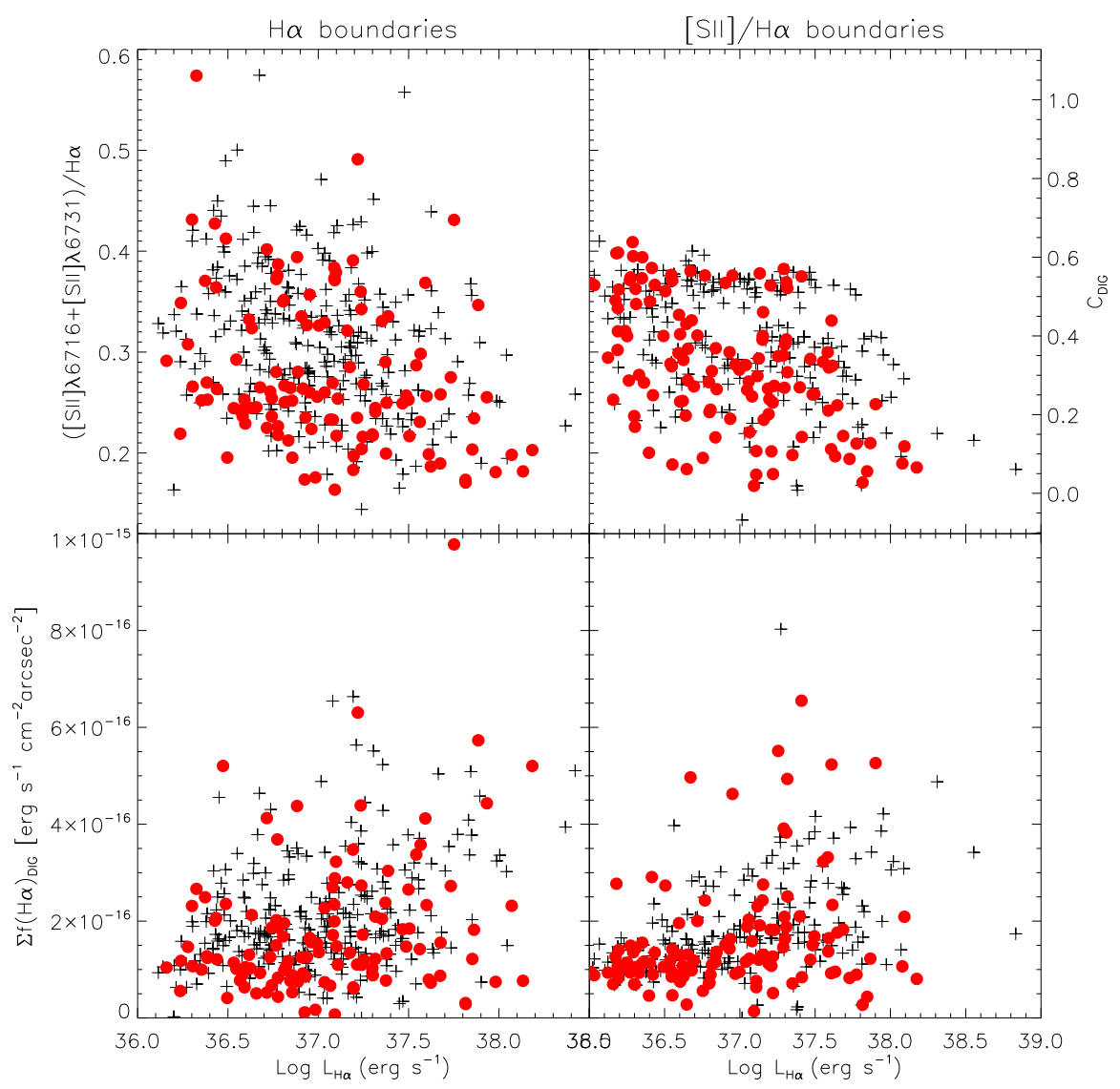

Figure 3. [S II] $/ \mathrm{H} \alpha$ is used to constrain DIG emission for the arm (black) and interarm (red) $\mathrm{H}$ II regions. $\mathrm{H}$ II regions are identified using either $\mathrm{H} \alpha$ emission (left) or $[\mathrm{S} \mathrm{II}] / \mathrm{H} \alpha$ line ratios (right). Top: [S II] $/ \mathrm{H} \alpha$ ratio as a function of luminosity of the $\mathrm{H}$ II region. The typical uncertainty is 0.04 . The corresponding diffuse gas fraction $\left(C_{\mathrm{DIG}}\right)$ is shown on the right axis. Interarm $\mathrm{H}$ II regions show ratios more consistent with pure star formation, while arm regions suffer from a larger diffuse gas contamination. Bottom: contamination of $\mathrm{H} \alpha$ surface brightness due to the diffuse gas background to each $\mathrm{H}$ II region as a function of the region's luminosity.

cannot distinguish between these two populations), but suffers from many biases. As our fields are centered on interarm regions, we miss the brightest $\mathrm{H}$ II regions in spiral arms. In addition, our $35 \mathrm{pc}$ resolution limits us to resolving the brightest regions $>10^{38} \mathrm{erg} \mathrm{s}^{-1}$ (Gutiérrez et al. 2011). Because of these biases we do not attempt to fit the luminosity function, but show our luminosity distribution in relation to the slope previously reported for NGC 628 (Kennicutt \& Hodge 1980). More extended spatial coverage is necessary to disentangle any changes between our novel IFU-based luminosity function and previous narrow-band imaging results.

We convert the size of each region, in pixels, to an equivalent radius and find $20 \mathrm{pc}<r_{\mathrm{eq}}<100 \mathrm{pc}$. The size of an $\mathrm{H}$ II region is related to its age (Whitmore et al. 2011), though we are resolving only the largest, and thus the oldest (6-8 Myr), of the $\mathrm{H}$ II regions, and in this regime the size has little sensitivity as a diagnostic of age. The same range of sizes is seen in both environments, with nearly half of regions unresolved $(r \sim 35 \mathrm{pc})$, though this is sensitive to both the method of identifying the region and the increased diffuse background on the spiral arms (see Section 4.1).

The metallicities of $\mathrm{H}$ II regions have a well established radial dependence (Zaritsky et al. 1994; Ho et al. 2015), but the $2 \mathrm{D}$ variations are less well explored. M101 has shown evidence for azimuthal variations in gas-phase metallicity (Kennicutt \& Garnett 1996; Li et al. 2013); however; Cedrés \& Cepa (2002) found no difference between arm and interarm metallicities. Conversely, in NGC 628 and NGC 6946, Cedrés et al. (2012) found evidence of $\mathrm{H}$ II regions with higher metallicity along the spiral arms. We calculate the gas-phase oxygen abundance using the strong line diagnostic of Dopita et al. (2016), which accounts for the ionization state without the necessity of the [O II] $\lambda 3727$ doublet. Results are similar using the $N 2$ diagnostic of Pettini \& Pagel (2004). Linear fits to both populations agree within the uncertainties of the fit, and are consistent with the gradient measured using temperaturesensitive methods (Berg et al. 2015). For the H II regions in spiral arms we fit the radial metallicity gradient as

$$
\begin{aligned}
12+\log (\mathrm{O} / \mathrm{H})= & (8.790 \pm 0.017)+(-0.558 \pm 0.064) \\
& \times R\left(\operatorname{dex} R_{25}^{-1}\right) .
\end{aligned}
$$

For the interarm $\mathrm{H}$ II regions we fit the radial metallicity gradient as

$$
\begin{aligned}
12+\log (\mathrm{O} / \mathrm{H})= & (8.780 \pm 0.022)+(-0.422 \pm 0.101) \\
& \times R\left(\operatorname{dex} R_{25}^{-1}\right) .
\end{aligned}
$$

$[\mathrm{O} \mathrm{III}] / \mathrm{H} \beta$, which correlates with the ionization parameter, is also known to vary radially (Rosales-Ortega et al. 2011). Little work has been done to investigate the environmental dependence of this parameter, but Cedrés \& Cepa (2002) observed no difference in this ratio between arm and interarm H II regions within M101. We observe no difference between the arm and 
interarm populations, and linear fits to both populations agree within the uncertainties of the fit.

\subsection{Diffuse Ionized Gas}

Not all $\mathrm{H} \alpha$ emission observed arises from $\mathrm{H}$ II regions. A large fraction of $\mathrm{H} \alpha$ emission in galaxies can arise from the DIG (see reviews by Mathis 2000 and Haffner et al. 2009). Quite different values for the diffuse fraction have been found for interarm regions, from $0 \%$ for NGC 247 (Ferguson et al. 1996) to $100 \%$ for the central regions of M51 (Blanc et al. 2009). Given the sparse distribution and lower luminosities of $\mathrm{HII}$ regions in the interarm regions and the low surface brightness of the DIG, the diffuse fraction may depend significantly on the resolution and sensitivity to surface brightness of the observations.

We quantify the total contribution from diffuse emission to the $\mathrm{H} \alpha$ flux in the arm and interarm by summing all flux in each environment, and comparing it to the total flux in each environment that is outside the identified $\mathrm{H}$ II regions. We measure a $(17 \pm 2) \%$ diffuse fraction in the arm, which rises to $(49 \pm 10) \%$ in the interarm. Close examination of the $\mathrm{H} \alpha$ images reveals that there is still some contribution from lowlevel interarm star formation to what we have identified as diffuse emission. Lowering the threshold for $\mathrm{H} \alpha$ surface brightness used in the Clumpfind algorithm to $15 \sigma$ $\left(7.5 \times 10^{-17} \mathrm{erg} \mathrm{s}^{-1} \mathrm{~cm}^{-2} \operatorname{arcsec}^{-2}\right)$, which more completely identifies discrete structures, results in a decrease in the interarm diffuse fraction to $(36 \pm 9) \%$, but also associates many extended DIG features in the arm with the large H II region complexes.

The $[\mathrm{S} \mathrm{II}] / \mathrm{H} \alpha$ line ratio correlates with diffuse gas fraction, showing a narrow range of low values for emission purely from $\mathrm{H}$ II regions, and increasing values depending on the relative flux contribution from the diffuse gas (Madsen et al. 2006; Blanc et al. 2009). We explore the line ratio ([S II] $\lambda 6716+$ $\lambda 6731) / \mathrm{H} \alpha$ for our $\mathrm{H}$ II regions and find a difference between the arm and interarm populations (Figure 3). The interarm regions span a narrower range of values and are generally more consistent with pure $\mathrm{H}$ II regions. The arm regions show an anticorrelation with the $\mathrm{H} \alpha$ luminosity, indicating a larger diffuse fraction in fainter $\mathrm{H}$ II regions. This was seen clearly in M51 by Blanc et al. (2009); however, the difference between our arm and interarm $\mathrm{H}$ II regions suggests differing levels of contamination for the two environments.

\section{DISCUSSION}

The combination of spectral diagnostics mapped at high spatial resolution enables us to investigate some of the assumptions made when isolating the $\mathrm{H} \alpha$ emission that is associated with star formation. We model the impact of DIG contamination within our H II regions, and explore the novel use of $[\mathrm{S} \mathrm{II}] / \mathrm{H} \alpha$ for identifying the boundaries of $\mathrm{H}$ II regions. Finally, we measure the impact that the DIG correction could have on studies of star formation.

\subsection{Diffuse Background within H II Regions and the Distribution of DIG}

Given the variation in $[\mathrm{S} \mathrm{II}] / \mathrm{H} \alpha$ seen between $\mathrm{H}$ II regions, we investigate the use of this ratio to measure the fraction of $\mathrm{H} \alpha$ emission due to DIG that contributes as a background to the $\mathrm{H}$ II regions. Following Blanc et al. (2009), we model the
Table 1

[S II] $/ \mathrm{H} \alpha$ Ratios for Arm and Interarm Regions

\begin{tabular}{lcc}
\hline \hline & {$[\mathrm{S} \mathrm{II}] / \mathrm{H} \alpha$} & $\begin{array}{c}f(\mathrm{H} \alpha)_{\mathrm{DIG}} \\
\left(\mathrm{erg} \mathrm{s}^{-1} \mathrm{~cm}^{-2} \operatorname{arcsec}^{-2}\right)\end{array}$ \\
\hline $\begin{array}{l}\text { H II regions } \\
\text { Arm }^{\mathrm{a}}\end{array}$ & 0.32 & $(1.78 \pm 0.10) \times 10^{-16}$ \\
Interarm $^{\mathrm{a}}$ & 0.26 & $(1.35 \pm 0.08) \times 10^{-16}$ \\
DIG & 0.47 & $(1.29 \pm 0.12) \times 10^{-16}$ \\
Arm $^{\mathrm{b}}$ & 0.54 & $(0.86 \pm 0.11) \times 10^{-16}$ \\
Interarm $^{\mathrm{b}}$ & & \\
\hline
\end{tabular}

Notes.

${ }^{\mathrm{a}}$ Median value found within $\mathrm{H}$ II regions; $f(\mathrm{H} \alpha)_{\mathrm{DIG}}$ calculated following Equation (3).

b Measured from integrated DIG spectrum.

$\mathrm{H} \alpha$ flux as arising from two sources,

$$
\begin{aligned}
f(\mathrm{H} \alpha) & =f(\mathrm{H} \alpha)_{\mathrm{H} \text { II }}+f(\mathrm{H} \alpha)_{\mathrm{DIG}} \\
& =C_{\mathrm{H} \text { II }} f(\mathrm{H} \alpha)+C_{\mathrm{DIG}} f(\mathrm{H} \alpha)
\end{aligned}
$$

where $C_{\mathrm{DIG}}$ is the fraction of total flux arising from the DIG and $C_{\mathrm{DIG}}=1-C_{\mathrm{H} \text { II }}$. The observed $[\mathrm{S} \mathrm{II}] / \mathrm{H} \alpha$ ratio is then

$$
\frac{[\mathrm{S} \mathrm{II}]}{\mathrm{H} \alpha}=C_{\mathrm{H} \mathrm{II}}\left(\frac{[\mathrm{S} \mathrm{II}]}{\mathrm{H} \alpha}\right)_{\mathrm{H} \mathrm{II}}+C_{\mathrm{DIG}}\left(\frac{[\mathrm{S} \mathrm{II}]}{\mathrm{H} \alpha}\right)_{\mathrm{DIG}} .
$$

We measure the typical $[\mathrm{S} \mathrm{II}] / \mathrm{H} \alpha$ ratio in the arm and interarm, taking the median value for the $\mathrm{H}$ II regions and using an integrated spectrum for diffuse regions (Table 1). ([S II]/ $\mathrm{H} \alpha)_{\text {DIG }}$ is similar between the arm and interarm, supporting our claim that we identify the bulk of the $\mathrm{H}$ II regions within both environments, and is systematically higher than $([\mathrm{S} \mathrm{II}] / \mathrm{H} \alpha)_{\mathrm{H} \text { II }}$.

The $[\mathrm{S} \mathrm{II}] / \mathrm{H} \alpha$ ratios within the $\mathrm{H}$ II regions show a range of values (Figure 3), with the interarm H II regions having consistently lower ratios. There are a number of possible explanations for this apart from a difference in DIG contribution. $[\mathrm{S} \mathrm{II}] / \mathrm{H} \alpha$ decreases with increasing ionization parameter, decreasing metallicity, decreasing spectral hardness (age), and increasing escape fraction of ionizing radiation. We have constrained the first two conditions and found no difference with environment (Section 3.1), but have no way to constrain the last two conditions. These emission lines are near enough in wavelength that the effect of dust reddening is negligible.

Assuming the variations in $[\mathrm{S} \mathrm{II}] / \mathrm{H} \alpha$ are due to DIG contamination, we quantify this within each H II region. Following Equation (4), we calculate $C_{\text {DIG }}$, assuming ([S II]/ $\mathrm{H} \alpha)_{\mathrm{H} \mathrm{II}}=0.16$, the minimum value for our sample, and ([S II]/ $\mathrm{H} \alpha)_{\text {DIG }}=0.51$, the average from Table 1 . If instead we use the single [S II] $\lambda 6716$ line, as in Madsen et al. (2006), we obtain similar results and a ratio $([\mathrm{S} \mathrm{II}] \lambda 6716 / \mathrm{H} \alpha)_{\mathrm{H} \text { II }}=0.10$ that is consistent with HII regions in the Milky Way (Madsen et al. 2006). We find a median diffuse fraction that is nearly twice as high in the $\mathrm{H}$ II regions on the arm as in interarm regions $((44 \pm 11) \%$ versus $(28 \pm 10) \%)$ and an anticorrelation with $L_{\mathrm{H} \alpha}$ (Figure 3).

We then calculate $f(\mathrm{H} \alpha)_{\text {DIG }}$, and convert this to a DIG surface brightness on the basis of the spatial extent. For the DIG regions, we use an integrated spectrum to directly measure the extinction-corrected $\mathrm{H} \alpha$ surface brightness. All values are listed in Table 1. We observe a higher DIG surface brightness in the arms than in the interarm, and a higher contribution to the DIG surface brightness within the $\mathrm{H}$ II regions than in the 
surrounding area. This is expected if continuum radiation that has leaked from $\mathrm{H}$ II regions contributes significantly to photoionizing the DIG (Zurita et al. 2000), and it suggests that we may be systematically underestimating the ionizing radiation emitted by the $\mathrm{H}$ II region because we have not accounted for these leaked photons.

This difference in DIG background level could bias the measured sizes on spiral arms to be larger than $\mathrm{H}$ II regions of similar size in the interarm when using a threshold for the $\mathrm{H} \alpha$ surface brightness. This could also bias the luminosity limit for detecting $\mathrm{H}$ II regions within the arm and interarm.

\subsection{Identification of H II Regions from Physical Conditions}

The multiple line maps obtained from our optical IFU spectroscopy provide a novel opportunity to revisit established methods for identifying the boundaries of $\mathrm{H}$ II regions. The $[\mathrm{S} \mathrm{II}] / \mathrm{H} \alpha$ line ratio provides a method that is based on variations in temperature rather than in line intensity. We find that the H II regions generally cover the same extent, and we more easily identify fainter regions but are less successful at dividing clustered regions (Figure 1). The boundaries of the region are more irregular due to the lower intensity of the [S II] line.

Our catalog of $\mathrm{H}$ II regions identified by [S II] $/ \mathrm{H} \alpha$ results in a realistic range of luminosities and sizes (Figure 2). The arm/ interarm populations appear more similar in their size distribution than $\mathrm{HII}$ regions defined by the $\mathrm{H} \alpha$ intensity alone. The diffuse fractions in the arm and interarm for these $[\mathrm{S} \mathrm{II}] / \mathrm{H} \alpha$ boundaries are $(21 \pm 2) \%$ and $(55 \pm 11) \%$, respectively. This is consistent with what was found with the $\mathrm{H} \alpha$ boundaries, supporting our claim that both methods recover similar $\mathrm{H}$ II regions.

Within the $\mathrm{H}$ II regions, the $[\mathrm{S} \mathrm{II}] / \mathrm{H} \alpha$ line ratios are restricted, by definition, to lower values (Figure 3). We still see on average lower DIG fractions for interarm H II regions, suggesting that this is not just an $\mathrm{H} \alpha$ boundary issue biased by increased DIG background on the arms. The median diffuse background surface brightness within $\mathrm{H}$ II regions is similar $\left(1.62 \times 10^{-16}\right.$ and $1.19 \times 10^{-16} \mathrm{erg} \mathrm{s}^{-1} \mathrm{~cm}^{-2} \operatorname{arcsec}^{-2}$ in the arm and interarm, respectively) and follows the same trend with environment.

As maps of this line ratio will be available for most optical IFU studies, it provides a new method for distinguishing the boundaries of $\mathrm{H}$ II regions, and will be essential for constructing maps of $\mathrm{H} \alpha$ line emission cleaned of all contaminants for use in studies of star formation.

\subsection{Kennicutt-Schmidt Law at 35 pc and the Role of Spatial Averaging}

We explore variations in the Kennicutt-Schmidt law (Schmidt 1959; Kennicutt 1989, 1998) to understand how the efficiency of star formation changes with galactic environment. Brinchmann et al. (2013) showed that optical spectroscopy alone can be used to estimate the total gas mass surface density within a factor of two on kiloparsec scales, and we use here a simplified model based on recent empirical results on smaller spatial scales.

We use dust attenuation as measured from the $\mathrm{H} \alpha / \mathrm{H} \beta$ Balmer line decrement, assuming a dust extinction law like that of the Milky Way (Cardelli et al. 1989) and $R_{V}=3.1$, to estimate the dust mass surface density (see Kreckel et al. 2013).
Assuming a fixed ratio of dust to gas of 0.01 (Sandstrom et al. 2013), we convert this to a total gas surface density. Note that this method suffers a bias in surface brightness, because we can measure only high extinction (and therefore high gas mass) on $\mathrm{H}$ II regions of high surface brightness (and therefore high SFR). We use the extinction-corrected $\mathrm{H} \alpha$ luminosity and sizes of $\mathrm{H}$ II regions to calculate the SFR surface density (Kennicutt 1998). Note that, due to its stochastic nature, the SFR is probably not well defined for these $\sim 35$ pc spatial scales when selecting individual $\mathrm{H}$ II regions. We are also potentially systematically underestimating our SFR because we have not accounted for leaked photons that contribute to ionizing the DIG.

In comparison with previous studies of star formation using total gas on sub-kiloparsec scales (Kennicutt et al. 2007; Bigiel et al. 2008), we see good agreement with the established relations, considering the significant assumptions that go into our model (Figure 4). Calculating a gas depletion time, we find similar values for arm and interarm of $2 \times 10^{9} \mathrm{yr}$. We see no difference between arm and interarm regions. Foyle et al. (2010) also found no evidence for differing gas depletion times on scales of $250-600 \mathrm{pc}$. Other nearby galaxies show very different results. NGC 6946, a more flocculent spiral galaxy, shows increased star formation efficiency (lower depletion times) on spiral arms (Rebolledo et al. 2012), and M51 exhibits a large scatter in the star formation efficiency along its spiral arms (Meidt et al. 2013).

The gas depletion times we estimate here are significantly longer than the lifetimes typically measured for GMCs (Meidt et al. 2015). However, if clouds are destroyed via shear or feedback on shorter timescales (20-30 Myr) they must also be created in order to maintain the present rate of star formation and the present efficiency. This is consistent with a constant cycling of molecular material between bound, cloud-like objects and a more diffuse state (Meidt et al. 2015).

We observe good agreement with the results of Schruba et al. (2010) when they explored the role that spatial resolution plays in calculated depletion times. We are targeting star-forming regions, so increasing our aperture to cover larger spatial scales will necessarily introduce regions without ongoing star formation, and lower the SFR surface density. Spatial averaging over scales of $500 \mathrm{pc}$ results in longer depletion times (Figure 4, left). However, given that we should be biasing our results to high SFRs because we are purposefully selecting only H II regions, our gas depletion times are an order of magnitude longer than those found by Schruba et al. (2010) on scales of $75 \mathrm{pc}$ with similar biases in M33. Direct observations of the molecular gas are necessary to confirm the environmental trends that are suggested here in NGC 628.

Including a correction for the diffuse gas contamination of each $\mathrm{H}$ II region results in decreased $\mathrm{H} \alpha$ luminosities and hence lower SFRs and longer depletion times of $\sim 1$ Gyr (Figure 4, right). This is on average a $62 \%(40 \%)$ increase in the spiral arms (interarms), and anticorrelates with the luminosity of $\mathrm{H}$ II regions, thus steepening the Kennicutt-Schmidt law. Given the relatively large diffuse contribution, particularly within spiral arms, this correction factor must be taken into account when calculating $\mathrm{H} \alpha$ SFRs. For a global spectrum, this DIG correction results in a $56 \%$ change, a significant correction to consider for unresolved (high-redshift) sources. 

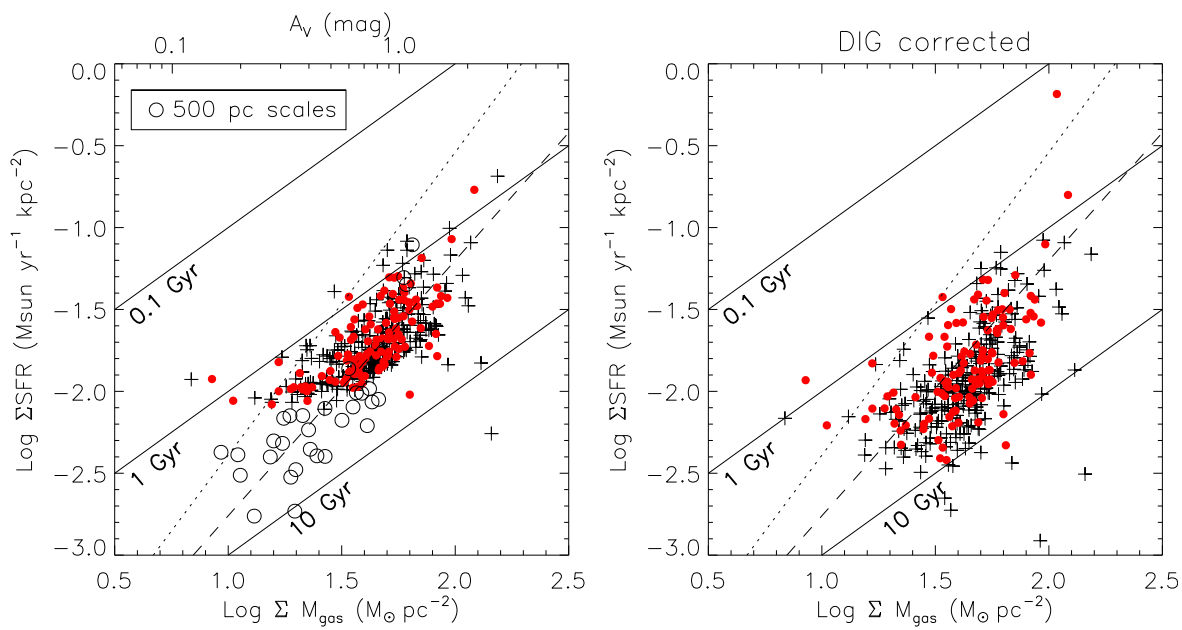

Figure 4. Total gas Kennicutt-Schmidt law for gas on scales of $35 \mathrm{pc}$. Constant depletion times of $10^{8}, 10^{9}$, and $10^{10} \mathrm{yr}$ are shown as solid lines. Left: arm (black) and interarm (red) H II regions are consistent with Kennicutt et al. (2007, dashed) and Bigiel et al. (2008, dotted), though those studies were limited to larger spatial scales $(350-500 \mathrm{pc})$. Binning our regions to scales of $500 \mathrm{pc}$ (open circles) results in longer depletion times. Right: including a DIG correction shifts all regions to longer depletion times by $40 \%$ for the interarm and $62 \%$ for the arm, and steepens the relation.

\section{IMPLICATIONS}

Recent results have shown that there is no "universal" GMC, but that cloud properties vary significantly between arm, interarm, upstream, and downstream regions (Colombo et al. 2014). However, our study suggests that while the properties of the birthplace may change, the resulting H II regions appear largely indistinguishable. This supports recent statistical studies comparing the luminosity functions of $\mathrm{H}$ II regions in spiral arm and interarm environments, which largely show no difference (Scoville et al. 2001; Azimlu et al. 2011; Gutiérrez et al. 2011).

GMCs in spiral arms are typically more massive, and are therefore expected to form a larger number of massive stars, inject more mechanical energy, and ionize the gas more strongly. Given that we see no difference between the ionization states of arm and interarm H II regions (Figure 2), either the efficiency with which they form is lower in the arms or the gas in the arms is less affected by feedback (more porous or fragmented).

Alternatively, our result might reflect the fact that the more extended envelope of the GMC is sensitive to local environmental effects while the star formation process that occurs in the dense clumps and cores is largely decoupled from the cloud as a whole. While properties of $\mathrm{CO}$ clouds may regulate the number of dense clumps that form, the process by which the densest gas forms stars (and creates an H II region) is universal. This is consistent with findings that the $\mathrm{HCN}$ gas surface density is linear with $\Sigma_{\mathrm{SFR}}$ and largely independent of local conditions, while the $\mathrm{CO}$ gas surface density shows greater variation (Gao \& Solomon 2004; Usero et al. 2015; Bigiel et al. 2016).

A future resolved, cloud-scale study of the molecular and dense gas in NGC 628 will test these scenarios directly.

We would like to thank the referee for helpful comments. We thank Kelley Foyle for providing arm/interarm masks, and I-Ting Ho for early use of LZIFU. K.K. acknowledges grant KR 4598/1-2 and S.M. acknowledges grant SCHI 536/7-1 from the DFG Priority Program 1573. G.B. is supported by CONICYT/FONDECYT, Programa de Iniciacion, Folio
11150220. B.G. gratefully acknowledges the support of the Australian Research Council as the recipient of a Future Fellowship (FT140101202). A.H. acknowledges support from the Centre National d'Etudes Spatiales (CNES). Based on observations made with ESO Telescopes at the La Silla Paranal Observatory under programme ID 094.C-0623 and ID 095. C-0473.

\section{REFERENCES}

Azimlu, M., Marciniak, R., \& Barmby, P. 2011, AJ, 142, 139 Bacon, R., Accardo, M., Adjali, L., et al. 2010, Proc. SPIE, 7735, 773508

Berg, D. A., Skillman, E. D., Croxall, K. V., et al. 2015, ApJ, 806, 16 Bigiel, F., Leroy, A., Walter, F., et al. 2008, AJ, 136, 2846

Bigiel, F., Leroy, A. K., Jiménez-Donaire, M. J., et al. 2016, ApJL, 822, L26 Blanc, G. A., Heiderman, A., Gebhardt, K., Evans, N. J., II, \& Adams, J. 2009, ApJ, 704, 842

Brinchmann, J., Charlot, S., Kauffmann, G., et al. 2013, MNRAS, 432, 2112 Bryant, J. J., Owers, M. S., Robotham, A. S. G., et al. 2015, MNRAS, 447, 2857

Bundy, K., Bershady, M. A., Law, D. R., et al. 2015, ApJ, 798, 7

Calzetti, D., Wu, S.-Y., Hong, S., et al. 2010, ApJ, 714, 1256

Cardelli, J. A., Clayton, G. C., \& Mathis, J. S. 1989, ApJ, 345, 245

Cedrés, B., \& Cepa, J. 2002, A\&A, 391, 809

Cedrés, B., Cepa, J., Bongiovanni, Á, et al. 2012, A\&A, 545, A43

Colombo, D., Meidt, S. E., Schinnerer, E., et al. 2014, ApJ, 784, 4

Dobbs, C. L., Bonnell, I. A., \& Pringle, J. E. 2006, MNRAS, 371, 1663

Dopita, M. A., Kewley, L. J., Sutherland, R. S., \& Nicholls, D. C. 2016, Ap\&SS, 361, 61

Ferguson, A. M. N., Wyse, R. F. G., Gallagher, J. S., III, \& Hunter, D. A. 1996, AJ, 111, 2265

Foyle, K., Rix, H.-W., Walter, F., \& Leroy, A. K. 2010, ApJ, 725, 534

Gao, Y., \& Solomon, P. M. 2004, ApJ, 606, 271

Greenawalt, B., Walterbos, R. A. M., Thilker, D., \& Hoopes, C. G. 1998, ApJ, 506, 135

Gutiérrez, L., Beckman, J. E., \& Buenrostro, V. 2011, AJ, 141, 113

Haffner, L. M., Dettmar, R.-J., Beckman, J. E., et al. 2009, RvMP, 81, 969

Ho, I.-T., Kudritzki, R.-P., Kewley, L. J., et al. 2015, MNRAS, 448, 2030

Ho, I.-T., Medling, A. M., Groves, B., et al. 2016, arXiv:1607.06561

Zurita, A., Rozas, M., \& Beckman, J. E. 2000, A\&A, 363, 9

Kennicutt, R. C., Calzetti, D., Aniano, G., et al. 2011, PASP, 123, 1347

Kennicutt, R. C., \& Hodge, P. W. 1980, ApJ, 241, 573

Kennicutt, R. C., Jr. 1989, ApJ, 344, 685

Kennicutt, R. C., Jr. 1998, ARA\&A, 36, 189

Kennicutt, R. C., Jr., Calzetti, D., Walter, F., et al. 2007, ApJ, 671, 333

Kennicutt, R. C., Jr., Edgar, B. K., \& Hodge, P. W. 1989, ApJ, 337, 761

Kennicutt, R. C., Jr., \& Garnett, D. R. 1996, ApJ, 456, 504

Knapen, J. H. 1998, MNRAS, 297, 255 
Knapen, J. H., Arnth-Jensen, N., Cepa, J., \& Beckman, J. E. 1993, AJ, 106, 56 Kreckel, K., Groves, B., Schinnerer, E., et al. 2013, ApJ, 771, 62 Lee, J. H., Hwang, N., \& Lee, M. G. 2011, ApJ, 735, 75

Leroy, A. K., Walter, F., Bigiel, F., et al. 2009, AJ, 137, 4670

Leroy, A. K., Walter, F., Sandstrom, K., et al. 2013, AJ, 146, 19

Li, Y., Bresolin, F., \& Kennicutt, R. C., Jr. 2013, ApJ, 766, 17

Madsen, G. J., Reynolds, R. J., \& Haffner, L. M. 2006, ApJ, 652, 401

Mathis, J. S. 2000, ApJ, 544, 347

Meidt, S. E., Hughes, A., Dobbs, C. L., et al. 2015, ApJ, 806, 72

Meidt, S. E., Schinnerer, E., García-Burillo, S., et al. 2013, ApJ, 779, 45

Oey, M. S., Meurer, G. R., Yelda, S., et al. 2007, ApJ, 661, 801

Pettini, M., \& Pagel, B. E. J. 2004, MNRAS, 348, L59

Rand, R. J. 1992, AJ, 103, 815

Rebolledo, D., Wong, T., Leroy, A., Koda, J., \& Donovan Meyer, J. 2012, ApJ, 757, 155

Rosales-Ortega, F. F., Díaz, A. I., Kennicutt, R. C., \& Sánchez, S. F. 2011, MNRAS, 415, 2439
Rozas, M., Beckman, J. E., \& Knapen, J. H. 1996, A\&A, 307, 735

Sánchez, S. F., Kennicutt, R. C., Gil de Paz, A., et al. 2012, A\&A, 538, A8

Sandstrom, K. M., Leroy, A. K., Walter, F., et al. 2013, ApJ, 777, 5

Schmidt, M. 1959, ApJ, 129, 243

Schruba, A., Leroy, A. K., Walter, F., Sandstrom, K., \& Rosolowsky, E. 2010, ApJ, 722, 1699

Scoville, N. Z., Polletta, M., Ewald, S., et al. 2001, AJ, 122, 3017

Skibba, R. A., Engelbracht, C. W., Dale, D., et al. 2011, ApJ, 738, 89

Thilker, D. A., Braun, R., \& Walterbos, R. A. M. 2000, AJ, 120, 3070

Usero, A., Leroy, A. K., Walter, F., et al. 2015, AJ, 150, 115

Vazdekis, A., Ricciardelli, E., Cenarro, A. J., et al. 2012, MNRAS, 424 157

Whitmore, B. C., Chandar, R., Kim, H., et al. 2011, ApJ, 729, 78

Williams, J. P., de Geus, E. J., \& Blitz, L. 1994, ApJ, 428, 693

Zaritsky, D., Kennicutt, R. C., Jr., \& Huchra, J. P. 1994, ApJ, 420, 87

Zurita, A., Rozas, M., \& Beckman, J. E. 2000, A\&A, 363, 9 